\title{
Kann Demokratie das Wachstum fördern?
}

\section{Till Nikolaus Folger}

\section{Relevanz}

Der Staat sind wir. Mit Demokratie lenken wir die Politik zu unserem Vorteil. Breiter Zugang zu Bildung steigert nicht nur die Produktivität, sondern fördert auch die demokratische Teilhabe und Kontrolle. Wirtschaftliche Reformen werden wahrscheinlicher, die den Nutzen für eine große Mehrheit anstatt des Vorteiles einer privilegierten Minderheit fördern. Doch die Demokratie ist nicht perfekt. Weltanschauliche Differenzen können den Konsens erschweren und wichtige Entscheidungen verhindern. Braucht es den aufgeklärten Autokraten, um nachhaltigen Wohlstand zu schaffen? Oder ist die Demokratie der verlässlichere Weg zu inklusivem Wachstum?

\section{Quelle}

Acemoglu Acemoglu, Daron, Suresh Naidu, Pascual Restrepo, James A. Robinson (2019), Democracy Does Cause Growth, Journal of Political Economy $127,47-100$.

Winston Churchill meinte einst, die Demokratie sei ,die schlechteste aller Regierungsformen - abgesehen von all den anderen Formen, die von Zeit zu Zeit ausprobiert worden sind“. Ist die Demokratie tatsächlich das politische Regime, das den Menschen den grössten Wohlstand beschert? China wird vielfach als undemokratisch gerügt, konnte jedoch über die letzten Jahrzehnte eine nie zuvor gesehene Wachstumsgeschichte schreiben. Auch die zunehmenden

T. N. Folger $(\bowtie)$

Universität St.Gallen, St.Gallen, Schweiz

E-Mail: till.folger2@student.unisg.ch 
populistischen, antidemokratischen Tendenzen in Europa und den USA werfen die Frage auf, ob Demokratie wirklich den besten Weg zu mehr Wachstum weist. Die Ökonomen Daron Acemoglu, Suresh Naidu, Pascual Restrepo und James A. Robinson von den Universitäten MIT, Columbia und Harvard untersuchen daher, ob die Demokratisierung einem Land tatsächlich mehr Wohlstand beschert. Der aktuelle Forschungsstand ist eher pessimistisch. Die Wissenschaftler meinen jedoch, dass die existierende Forschung zum Thema methodische Unzulänglichkeiten aufweist, z. B. im richtigen Messen der Demokratieniveaus.

Eine einfache Korrelation zwischen Demokratieniveau und Wachstum ist schnell berechnet. Die Forscher zeigen jedoch, dass der Effekt tatsächlich von der Demokratisierung ausgeht und das Wachstum ursächlich bedingt. Das Problem ist, dass auch historische und kulturelle Unterschiede sowohl das politische Regime als auch das Wachstum beeinflussen und somit den ursächlichen Einfluss verwaschen können. Oft lösen Krisen mit einem starken Rückgang des Pro-Kopf-Einkommens eine Demokratisierung aus, die dann wieder auf die Einkommensentwicklung zurückwirkt. Der Zusammenhang ist beidseitig und geht nicht nur in eine Richtung.

Für ihre Studie betrachten die Wissenschaftler 175 Länder über einen Zeitraum von 1960 bis 2010. Dabei unterscheiden sie zwischen „demokratischen“ und „nicht-demokratischen“ System. Die Daten zeigen eine deutliche Ausbreitung der Demokratie.

Das Demokratieniveau auf der Welt hat eindeutig zugenommen. Wurden 1960 31.5 Prozent der Länder als demokratisch eingestuft, waren es 2010 mit 64.1 Prozent mehr als doppelt so viele.

Die Forscher betrachten nicht nur permanente, sondern auch temporäre Systemwechsel. So hielt z. B. Argentinien 1973 erstmals seit zehn Jahren demokratische Wahlen ab. Nach einem Putsch nur drei Jahre später sollte es jedoch bis 1983 dauern, ehe Argentinien endgültig zur Demokratie wurde. In der Analyse gilt folglich nicht nur der Zeitraum ab 1983 als demokratisch, sondern auch jener zwischen 1973 und 1976.

Ein erster Blick auf die Daten verrät, dass Demokratien im Durchschnitt ein vierfach höheres Pro-Kopf-Einkommen haben und ihre Einwohner besser ausgebildet sind. Allerdings reicht eine solche Korrelation zwischen Demokratie und Einkommen nicht aus, um die Demokratisierung klar auf das Wachstum zurückzuführen. Das Forscherteam bedient sich insgesamt dreier ökonometrischer Analysen, um den Effekt der Demokratisierung auf den Wohlstand festzumachen. Alle drei Ansätze führen zu ähnlichen Erkenntnissen: Im Durchschnitt steigert Demokratisierung das Pro-Kopf-Einkommen, während eine Abkehr von der Demokratie einen Einkommensrückgang verursacht. 


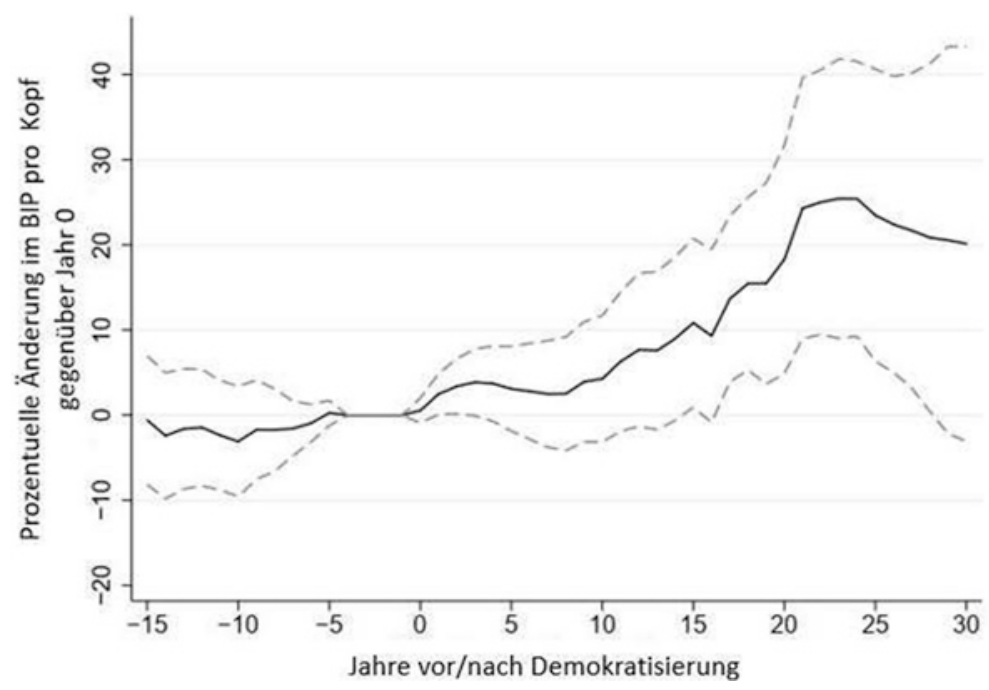

Abb. 1 Demokratisierung und Wohlstand. (Quelle: Acemoglu u. a. 2019)

Abb. 1 stellt die Einkommensentwicklung vor und nach dem Jahr Null, dem Zeitpunkt der Demokratisierung, dar. Die durchgezogene Linie zeigt die prozentuale Veränderung des Pro-Kopf-Einkommens im Vergleich zum Jahr der Demokratisierung und die gestrichelten Linien geben den Bereich an, in dem sich $95 \%$ der tatsächlichen Beobachtungen befinden. Während die Wissenschaftler vor dem Systemwechsel kaum Wachstum feststellen, schätzen sie das Pro-KopfEinkommen nach 20 bis 25 Jahren um etwa $24 \%$ höher als im Fall ohne Demokratisierung.

Demokratisierung verspricht langfristige Wohlstandsgewinne. Nach 20-25 Jahren erhöht sie das Pro-Kopf-Einkommen um rund ein Viertel.

Ein oft beobachtetes Phänomen im Demokratisierungsprozess sind regionalen Wellen, in denen dieser auftritt. Man denke an den Arabischen Frühling ab 2010 oder den Zerfall der Sowjetunion und ihrer Satellitenstaaten zu Beginn der 90er-Jahre. In den Daten zeigen sich solche „Ansteckungseffekte“ sehr deutlich. Sobald sich das erste Land in einer Region demokratisiert, dauert es meist nur wenige Jahre, bis der Anteil demokratischer Länder in der gesamten Region zum weltweiten Durchschnitt aufschliesst. Auch unter Berücksichtigung solcher 
regionalen Einflüsse auf die Wahrscheinlichkeit der Demokratisierung eines Landes schätzen die Forscher, dass Demokratie das Pro-Kopf-Einkommen langfristig um rund $26 \%$ steigert.

Welche spezifischen Mechanismen bestimmen, wie Demokratie das Wohlstandsniveau erhöht? Die empirischen Ergebnisse deuten darauf hin, dass die Demokratisierung eine Vielzahl von Faktoren fördert, welche Wachstum und Lebensstandard nachhaltig erhöhen. Wirtschaftliche Reformen werden wahrscheinlicher, die den Nutzen für eine grosse Mehrheit anstatt des Vorteiles einer privilegierten Minderheit fördern. Die Qualität des Bildungssystems verbessert sich, was den Anteil gut ausgebildeter Bürger steigert. Da die Forscher jedoch nicht gänzlich klären können, ob jene Kanäle tatsächlich Folgen des veränderten politischen Systems oder des erhöhten Wohlstands sind, bleiben diese Erkenntnisse etwas weniger eindeutig als die im Hauptteil der Analyse.

Demokratisierung steigert den Wohlstand sowohl in Ländern mit hohem, als auch in Ländern mit niedrigem Anfangseinkommen. Allerdings ist der Effekt auf das Wirtschaftswachstum stärker ausgeprägt, wenn ein grosser Teil der Bevölkerung weiterführende Schulen besucht hat.

Ein minimaler wirtschaftlicher Entwicklungsstand und eine gut ausgebildete Bevölkerung gelten als Voraussetzung für eine funktionierende Demokratie. Eine weit verbreitete Ansicht ist daher, dass Demokratisierung in schwach entwickelten Volkswirtschaften sogar nachteilig sein kann. Acemoglu und seine Kollegen finden jedoch keine Anhaltspunkte dafür, dass die Wohlstandseffekte der Demokratisierung systematisch vom Anfangsniveau des Einkommens abhängen. Allerdings verspricht die Demokratisierung stärkere Wohlstandsgewinne in Ländern mit einem hohen Bildungsstand.

Die Erkenntnisse dieser Arbeit zeigen, dass Demokratie nicht nur gesellschaftliche, sondern auch wirtschaftliche Vorteile mit sich bringt. Damit relativiert sie manche Zweifel an dieser Regierungsform. Trotz neuer und wichtiger Ergebnisse bleibt aber noch Raum für weitere Forschung. Demokratie kann mehr oder weniger weit gehen. Anstatt nur Demokratie und Nicht-Demokratie zu unterscheiden, könnte die Forschung z. B. auch den Einfluss eines unterschiedlichen Demokratisierungsgrades in verschiedenen politischen Systemen untersuchen. 
Open Access Dieses Kapitel wird unter der Creative Commons Namensnennung 4.0 International Lizenz (http://creativecommons.org/licenses/by/4.0/deed.de) veröffentlicht, welche die Nutzung, Vervielfältigung, Bearbeitung, Verbreitung und Wiedergabe in jeglichem Medium und Format erlaubt, sofern Sie den/die ursprünglichen Autor(en) und die Quelle ordnungsgemäß nennen, einen Link zur Creative Commons Lizenz beifügen und angeben, ob Änderungen vorgenommen wurden.

Die in diesem Kapitel enthaltenen Bilder und sonstiges Drittmaterial unterliegen ebenfalls der genannten Creative Commons Lizenz, sofern sich aus der Abbildungslegende nichts anderes ergibt. Sofern das betreffende Material nicht unter der genannten Creative Commons Lizenz steht und die betreffende Handlung nicht nach gesetzlichen Vorschriften erlaubt ist, ist für die oben aufgeführten Weiterverwendungen des Materials die Einwilligung des jeweiligen Rechteinhabers einzuholen.

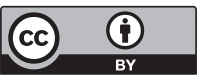

